\title{
Desarrollo Non Sancto. La religión como actor emergente en el debate global sobre el futuro del planeta
}

\author{
Adrián E. Beling y Julien Vanhulst (Coordinadores) \\ XXI Editores, México D.F. 2019, 349 p.
}

\author{
Daniel Duhart Smithson \\ Email: dduhart@gmail.com
}

\begin{abstract}
El lunes 14 de octubre de 2019 en la sala ‘Paulo Freire’ de la Universidad Academia de Humanismo Cristiano se realizó el primer lanzamiento oficial en Chile del libro «Desarrollo non Sancto. La religión como actor emergente en el debate global sobre el futuro del planeta», en el cual el autor de esta reseña participó como uno de los comentaristas. El enfoque de este libro se sitúa en el escenario de la 'crisis civilizatoria' por la que atraviesa América Latina, así como el mundo, reflejado principalmente en el imperativo ecológico que enfrenta la humanidad (cambio climático, calentamiento global, etc.), junto con las diversas problemáticas sociales, económicas y políticas interconectadas (creciente desigualdad, migración internacional, extensión de los niveles de pobreza y riqueza extremas, reducción de los niveles de seguridad y cohesión social, pérdida de gobernabilidad, la expansión de los conflictos raciales y nacionales, etc.). Sin embargo ninguno de los participantes en este lanzamiento se imaginaba que en sólo cuatro días después esta crisis ‘civilizatoria’ se visibilizaría de una manera tan dramática y amplia como ha sido con el estallido social que emergió en Chile desde el ya histórico viernes 18 de octubre de 2019, similar a los sucesos que se han expandido en diversos puntos del globo (Ecuador, Bolivia, Colombia, Hong Kong, Barcelona, etc.) y que continúan irrumpiendo el supuesto ‘orden’ del sistema de desarrollo global actual. A lo largo de Chile, y en varios de estos lugares, han surgido preguntas sobre cuáles pueden ser las fuentes éticas y gnositivas para una real y efectiva transición civilizatoria ante esta crisis que parece tocar las dimensiones más profundas del ser humano y la sociedad, con toda su complejidad; en este contexto, la lectura de este original y profundo libro parece ser no solo de una gran relevancia y necesidad, sino que casi una obligación dada la búsqueda cada vez más urgente de nuevas narrativas en un contexto de perplejidad creciente.
\end{abstract}

El libro «Desarrollo non Sancto. La religión como actor emergente en el debate global sobre el futuro del planeta» es una obra colectiva, coordinada por los sociológicos Adrian Beling y Julién Vanhulst, especialistas en el estudio del campo discursivo Latinoamericano sobre desarrollo sustentable, y en particular las nuevas líneas emergentes asociados al concepto de ‘buen vivir' o ‘vivir bien'. Es ya un gran logro y una primera gran razón para leer esta obra el reunir en un solo volumen a algunos de los representantes más destacados de la intelectualidad crítica Latinoamericana, tales Arturo Escobar, Enrique Leff, Eduardo Gudynas, Juan Carlos Scannone, Alberto Acosta, Gustavo Esteva, Guillermo Castro Herrera, Leonardo Boff (quien escribe uno de los prólogos), o a nivel global, como Wolfgang Sachs (autor del segundo prólogo) y Ulrich Brand, junto a otros importantes especialistas Latinoamericanos en el área de los estudios socio-ecológicos, culturales y territoriales, como Tatiana Roa Avendaño, María Luisa Eschenhagen, Raúl González Meyer, Daniela Escalona y Marilú Trautmann. Es original también la combinación de autores más representantes del área de los estudios sociales y medio ambientales junto con otros provenientes del mundo teológico y religioso, como Boff y Scannone desde América Latina, o Pirmin Spiegel en Europa, reflejo de otro de los logros de esta obra: generar un diálogo colectivo entre representantes del pensamiento crítico contemporáneo acerca de las posibles contribuciones de la religión en el debate sobre el futuro del planeta, una temática e interacción un tanto lejana dados los prejuicios 
y ‘tabúes' existentes desde el mundo progresista hacia la religión (muchas veces muy bien justificadas), aunque la tradición Latinoamericana de la teología de la liberación, entre otras, es una muestra de la conexión fructífera de este tipo de diálogos en el pasado.

El enfoque que los coordinadores tomaron para realizar este diálogo fue por medio de la discusión colectiva sobre la reciente encíclica del Papa Francisco, titulado «Laudato Si. Sobre el Cuidado de la Casa Común», que aunque no es la primera vez que se aborda el tema de la crisis ecológica desde una perspectiva religiosa o teológica (de hecho la llamada eco-teología tiene una gran presencia en América Latina desde al menos tres décadas, siendo uno de sus principales exponente justamente Leonardo Boff), esta encíclica ha tenido un importante impacto en el discurso público sobre desarrollo y medio ambiente, en especial en los sectores no confesionales. De hecho, como lo plantean los mismos coordinadores y autores de los prólogos, el lanzamiento de esta encíclica en octubre de 2015 coincidió con el surgimiento de otras dos declaraciones mundiales relevantes sobre el presente y futuro de la humanidad, como es la declaración de las Naciones Unidas sobre los Objetivos del Desarrollo Sostenible (ODS) y los Acuerdos para el Cambio Climático de la Conferencia de París (Cop21). El énfasis más radical y crítico del Laudato Si, un hecho sorpresivo para algunos sectores, ha llevado a una tensión creativa entre estos tres documentos, lo que es abordado por algunos de los autores de esta obra. Para lograr este diálogo y discusión colectiva, el libro está divido en tres grandes partes, cada uno compuesto por una serie de capítulos, además de los dos prólogos, una introducción y un epílogo escrito por los coordinadores, aproximándose al objeto de estudio desde los mismos discursos, para luego ver su potencial de sinergia con otros discursos de transición así como su relevancia en el campo de los debates del desarrollo. Uno de los elementos originales de este libro es que, junto con contar con capítulos en cada parte escritos por diferentes autores bajo la modalidad de 'ensayo', el último capítulo de cada parte consiste en un capítulo colectivo construido sobre la base de una serie de entrevistas por parte de los coordinadores con un grupo de autores, construyendo un diálogo a distancia entre éstos frente a un conjunto de preguntas comunes.

Para los coordinadores de la obra el escenario desde el cual inician esta conversación es, como decíamos más atrás, uno de crisis del desarrollo global. Esta crisis estaría asociada a lo que denominan la expansión de un 'modo de vida imperial', reflejado en un estilo de vida dominante asociado al consumismo materialista y competitivo del actual sistema mundial capitalista; un modo de vida que se sostendría sobre una suerte de 'autoengaño', una especie de simulación de acciones de mejora (acuerdos internacionales, reformas institucionales, etc.) pero que finalmente se traducen solamente en reformas paliativas, ocultando las causas más profundas de la crisis socio-ecológica (el llamado 'modo de vida imperial'). La pregunta que enfrentan los autores es cómo promover una transición socio-ecológica para superar esta crisis, y cuál podría ser el papel de la religión en este proceso, un actor usualmente olvidado en este campo discursivo y político. Justamente los discursos de transición buscarían promover «un cambio en las condiciones subjetivas y objetivas que mantienen la insustentabilidad» (p.30), en contraste con los discursos que mantendrían el statu quo. Para los coordinadores, la religión habría «... acompañado el devenir histórico de la humanidad desde sus inicios», influenciando significativamente «las maneras de sentir, pensar y actuar, abarcando todos los grandes temas de la vida individual y colectiva, incluida la relación entre los seres humanos y la naturaleza no humana» (p.19), y actuando principalmente en tres planos: «imaginario cultural, praxis social e institucional» (p.20). Por ello no tendría sentido dejar fuera a un actor tan relevante de la historia de la humanidad. Sin embargo, cómo y de qué manera debería darse este rol, y finalmente qué estamos entendiendo sobre religión en la modernidad contemporánea, son temas que requieren un análisis profundo, una de las razones de la relevancia de leer un libro como este en la coyuntura mundial actual.

El libro introduce esta discusión con la primera parte, bajo el título «El llamado a construir una 'ecología integral'. Nueva narrativa eclesial en el contexto de crisis civilizatoria», que se centra en la inserción de lo que los autores denominan la narrativa religiosa de una 'ecología integral' en los debates globales sobre y sustentabilidad, y sus expresiones latinoamericanas. El primer autor que contribuye un capítulo a esta sección es Enrique Leff, quien realiza un agudo análisis sobre algunos de los elementos centrales de la mencionada encíclica, y sus conexiones con los discursos críticos latinoamericanos, abriendo un diálogo entre la encíclica y lo que Leff denomina la necesidad de una 'racionalidad ambiental', y en especial su centralidad en la reproducción de la vida y no del capital. El segundo capítulo es escrito por Myriam Lang, quien realiza un análisis profundo de las bases históricas de la crisis civilizatoria actual, analizando los elementos comunes de los diferentes modelos societales que han emergido desde la posguerra, reflejado en el discurso hegemónico común del desarrollo, incluyendo los 
recientes experimentos del llamado 'socialismo del siglo XXI', con un énfasis particular en Ecuador. Finalmente el tercer capítulo presenta el primer diálogo a distancia entre Guillermo Castro Herrera, Arturo Escobar, Gustavo Esteva, Eduardo Gudynas, Tatiana Roa Avendaño y Juan Carlos Scannone, abordando tres preguntas acerca de la emergencia de esta nueva narrativa religiosa de la ecología integral en los debates contemporáneos sobre desarrollo y sustentabilidad. La segunda parte del libro se titula «Las sinergias con otros discursos de transición», explorando las posibilidades de conexión entre esta emergente narrativa religiosa con otros discursos que propician una ‘Gran transición' socio-ecológica, tales como el decrecimiento, el postextractivismo y el buen vivir. En el capítulo 4 Ulrich Brand analiza los potenciales de sinergia entre los discursos del postextractivismo y el poscrecimiento, del sur y norte global respectivamente, así como entre éstos y el rol que podría caber a la religión en este contexto. En el capítulo 5 María Luisa Eshenhagen, en uno de los capítulos más originales del libro, explora las posibilidades de diálogo entre el summa qamaña aymara con el budismo, relevando sus disposiciones comunes para superar las inclinaciones humanas de la avaricia y el apego que sostendrían en gran parte el denominado 'modo de vida imperial' del consumismo capitalista, y sus consecuencias ecológicas. En el capítulo 6 Alberto Acosta presenta al ‘buen vivir' latinoamericano como una alternativa el 'maldesarrollo' del sistema mundial actual, relevando algunas de las sinergias entre este discurso de base indígena con el Laudato Si. El capítulo 7 presenta nuevamente la modalidad de entrevista colectiva y a distancia entre el mencionado grupo de autores, abordando en esta ocasión dos preguntas que buscan explorar la cercanía o distancia y los posibles puntos de acople entre la encíclica y otras propuestas para un cambio global hacia la sustentabilidad. Finalmente la tercera parte del libro, titulada «La religión como agente de transformación socioecológica: actualidad, perspectivas y alianzas», presenta algunas experiencias prácticas de cómo la religión podría constituir un actor en el debate sobre el futuro del planeta, tanto desde la perspectiva del impulso de una agenda de transformación desde los actores religiosos, como su articulación con otros entes públicos y privados, y a diferente nivel. En este camino, en el capítulo 8 Pirmin Spiegel presenta sus reflexiones sobre el rol de la religión en este contexto a partir de su experiencia como director de Misereor, la mayor organización de la cooperación católica para el desarrollo en todo el mundo, analizando tanto aspectos conceptuales así como una serie de experiencias prácticas que esta organización estaría impulsando en diferentes regiones del globo. En el capítulo 9 los chilenos Raúl González, Daniela Escalona y Marilú Trautmann analizan tanto los desafíos como los potenciales de sinergia y alianza entre movimientos de base ecológica y los actores religiosos, con un énfasis particular en los enfoques territoriales, dado la alta presencia de conflictos ambientales y la emergencia de diversos actores en las interfaces socioculturales en este tipo de contextos en Latinoamérica. Finalmente el capítulo 10 presenta el último capítulo de diálogo colectivo a distancia entre el grupo de autores entrevistados, esta vez en torno al papel de los actores religiosos como parte de una red de agentes transformadores.

Como se podrá observar por la breve descripción de los capítulos presentada más arriba, aunque siguen una secuencia lógica éstos abarcan una gran diversidad de contenidos y temáticas; sin embargo se pueden identificar algunos ejes comunes: varios de los autores relevan en la emergente narrativa religiosa de 'ecología integral' una relación entre la espiritualidad y la transformación ecológica, en cuanto a que sitúa a la religión como fuente de motivación para transformaciones personales y sociales en la superación de un modelo de sociedad basado en la avaricia, el consumo, el apego, la vanagloria, etc.; en este sentido, la religión podría verse como factor para una especie de 'conversión ecológica'. Otro elemento común que emerge del análisis de la encíclica y otros conceptos del campo interreligioso es el rechazo a la reducción de la economía y la tecnología como fines en sí mismos, re-articulándolos en cuanto medios para el logro del 'honor’ y dignidad humanos, y en este sentido fuente de bases civilizatorias alternativas al sistema moderno en crisis. Otra clave de lectura que emerge de estos capítulos es la conexión entre la espiritualidad y la 'tierra', uno de los temas más presentes en el Laudato Si, en una suerte de 'coqueteo' con las visiones de los pueblos originarios sobre la naturaleza como dotada de vida, similar a la ética de Francisco de Asís y su hermandad con los animales y la naturaleza, trascendiendo en alguna medida el llamado antropocentrismo del cual ha sido acusado la Iglesia Católica y otras expresiones cristianas. En este sentido, el diálogo y la cooperación interreligiosa emergen como una estrategia de transformación profunda, como se destaca en el capítulo sobre las conexiones entre el summa qamaña y el budismo. Otros autores destacan las conexiones discursivas y programáticas del contenido del Laudato Si con los movimientos de base y populares que luchan frente a los diversos conflictos ambientales que se expanden en el modelo de desarrollo global actual, y el rol a la vez liberador y articulador que sus conceptos entregarían. Otros relevan a la religión como actor institucional en la aplicación práctica de programas e iniciativas de transformación en comunidades y territorios en crisis, destacando por ejemplo las posibilidades de la religión 
como actor clave a través de sus redes de universidades y centros educativos, en cuanto actor en la sociedad civil, para una labor de investigación, sistematización, diálogo y acción concreta. Por último, emerge también por un lado una demanda desde varios autores, como Escobar, Gudynas y Esteva, sobre la necesidad de los actores religiosos de estar atentos a los elementos opresores que aún perviven en varias de sus estructuras organizacionales y de pensamiento, y que fácilmente podrían contribuir más bien a la mantención del 'modo de vida imperial' que sustenta la insustentabilidad actual; pero por el otro lado, celebran también la radicalidad del Laudato Si y otros documentos religiosos similares, y las tensiones y complementariedades que pueden identificarse entre éstos y otros documentos institucionales globales de gran relevancia. De hecho, como señalan los coordinadores de la obra en su epílogo, «El desarrollo se convierte, así, en una quimera; en un objetivo societal non sancto, deshonesto, que conlleva inherentemente la explotación de nuestra 'hermana y Madre Tierra' y de los pobres del mundo. Francisco lo subraya: 'Una estrategia de cambio real exige repensar la totalidad de los procesos, ya que no basta con incluir consideraciones ecológicas superficiales mientras no se cuestione la lógica subyacente en la cultura actual» (ls §197)» (p.333).

La pregunta que emerge es cómo ampliar esta discusión y proceso reflexivo para una mayor traducción en acción de este contenido que emerge de esta nueva narrativa religiosa, cómo ampliar el diálogo con los aportes de otras comunidades y religiones , y cómo llevarlo a una aplicación que apele a la transformación al mismo tiempo que genere alianzas, superando el ‘engaño' del modelo actual e impulsándolo de forma constructiva y esperanzadora, facilitando el proceso de aprendizaje que requiere el largo proceso de erección de una civilización planetaria; las transiciones civilizatorias son procesos largos y complejos, y no se pueden tomar atajos. Este libro es un primer gran paso en este camino, señalando varios ejemplos prácticos así como haciendo referencia a procesos en marcha a través de discusiones más conceptuales presentes en varios capítulos, constituyendo un aporte fundamental para este emergente campo de investigación y acción. Se podría decir que es una obra fundadora de en América Latina para un nuevo enfoque desde la sociología para el estudio de la religión desde la perspectiva de la transformación socio-ecológica y civilizatoria, levantando una serie de líneas o áreas de pesquisa que requieren un análisis continuado y cada vez más profundo. En esta misma línea, y como una contribución a este programa de investigación emergente, en una investigación reciente el autor de esta reseña ha identificado un contenido espiritual emergente en el campo de los discursos latinoamericanos de transición civilizatoria, en muchos casos expresado en otras obras de los algunos de los mismos intelectuales que participan de esta obra, el cual podría contener un potencial de semántica novedosa para la continua pregunta moderna sobre la construcción del orden social deseado (Duhart, 2019).

Parece existir una demanda de fuentes de certidumbre para hacer frente a la naturaleza incierta de la modernidad contemporánea, y la búsqueda de narrativas que puedan superar las maneras dicotómicas que han surgido en el pasado para expresar las relaciones entre elementos como lo material y lo espiritual, la ciencia y la religión, el ser humano y la naturaleza, lo universal y lo particular, lo lineal y lo circular, el mejoramiento y la regeneración, el crecimiento o el decrecimiento, etc., en una serie de tensiones creativas que exploran formas más interdependientes, holistas y complementarias para aproximarse a la realidad. En este sentido, una serie de nociones de lo espiritual que lo asocian a la acción, a la reflexividad, a la naturaleza, a la ciencia, a la complementariedad, a la complejidad, a la capacidad de aprender, a una forma más ampliada de racionalidad, entre otras, serían expresiones de esta semántica que por un lado exige un rol activo a la religión en cuanto fuente de percepciones sobre el mundo (como un sistema de conocimiento y práctica), al mismo tiempo que demanda expresiones más ‘maduras’ o nutridas acordes a las necesidad de una civilización y paradigma ecológico mundial emergente, libre de dogmas y jerarquías opresoras. ¿Qué tipo de experiencias práctica podrían reflejar de manera concreta este tipo de dualidades complementarias?

En esta misma investigación, el autor relevó algunas de las capacidades que emergen desde experiencias concretas de articular contenidos espirituales y materiales, en vez de contraponerlos o concebirlos en competencia, que ayudan a comunidades a explorar un sendero de bienestar sobre la base de un diálogo de saberes entre contenidos espirituales, científicos modernos, y la experiencia de otros pueblos, permitiendo construir un modelo de ‘mejoramiento’ y 'regeneración’ más armónico en su territorio (Duhart, 2019, p.301). Como reafirma la lectura de este valioso libro, el estudio sistemático y más amplio de este tipo de experiencias, la diseminación de sus aprendizajes, y la promoción y profundización de los alcances de sus hallazgos, sería el objeto de investigación de este nuevo campo de la sociología, junto con el desarrollo conceptual y teórico que emerge a partir de 
estas reflexiones, lo que podría profundizarse y ampliarse en un segundo volumen de esta obra, que dejamos aquí como una sugerencia a los coordinadores.

Esto se conecta finalmente, y para ir terminando esta reseña, con una de las afirmaciones más relevantes de los coordinadores presentada en el epilogo del libro, que tiene relación con que la crítica del modelo global de desarrollo no implica abandonar el horizonte normativo del mejoramiento de la realidad humana, sino que más bien su refinamiento y redefinición bajo nociones más amplias sobre la relación del ser humano consigo mismo, con otros seres humanos, y con la naturaleza, o como diría Hartmut Rosa en clave sociológica, entre los mundos subjetivo, intersubjetivo y objetivo (Rosa, 2015), donde de acuerdo con este libro la religión tendría mucho para contribuir en la construcción de grados crecientes de 'resonancia' entre éstos para un proceso de transición civilizatoria.

«Así, la crítica al desarrollo aquí propuesta no implica, en modo alguno, abandonar de plano un ideal de mejoramiento de la humanidad, sino la necesidad de cuestionar de manera fundamental los supuestos naturalizados respecto del significado preciso y las declinaciones prácticas de dicho ideal... La deconstrucción crítica de esta perversión del ideal de mejoramiento de la humanidad aparece, por tanto, como precondición para rehabilitar vías alternativas para construir otro mundo, un mundo que amplíe y profundice la libertad y autonomía creativa en armonía con el orden dado del cosmos, un mundo en el que los seres humanos se asumen parte de la red más amplia de la vida y de la dinámica planetaria, asumiendo a la Tierra como su ‘casa común’» (p.331).

\section{Referencias}

Duhart, Daniel (2019). ¿Descentrando lo material? El contenido espiritual en los discursos latinoamericanos de transición civilizatoria como semánticas emergentes para la construcción del orden social deseado. Tesis Doctorado de Sociología, Universidad Alberto Hurtado: Santiago.

Rosa, Hartmut (2015). Escalation: The Crisis of Dynamic Stabilisation and the Prospect of Resonance. En Dörre, Klaus, Lessenhich, Stephan y Rosa, Hartmut (Eds.), Sociology, Capitalism, Critique (pp. 322-347). Londres: Verso. 